\title{
Association of nutritional status and functional capacity in gastrointestinal cancer patients
}

\author{
Elizabeth Pérez-Cruz and Christian Patricio Camacho-Limas \\ Nutritional Support and Metabolism Unit, Hospital Juárez de México, Mexico City, Mexico
}

\begin{abstract}
Objective: To determine the nutritional status and its association with functional capacity in patients with digestive tract cancer. Methods: We retrospectively studied all adult patients hospitalized who were diagnosed as having a cancer of the digestive tract. Nutritional status and functional capacity were assessed. Descriptive statistic and odds ratio were used to determine the association in SPSS 14.0. Results: 57 patients were included, $96 \%$ had weight loss. Using subjective global assessment (SGA) as a method of screening, $82.5 \%$ of the patients were found malnutrition and by biochemical and immunological test $82 \%$ and $65 \%$ respectively. Functional capacity was assessed by Karnofsky index, finding that $75.5 \%$ of the patients have some activity limitation. Results show an association between malnutrition by SGA and limitation in functional capacity $(\chi 2=1.56$; $p=0.212$; OR: 2.46; 95\% confidence interval [95\% Cl]: 0.581-10.465). In addition, we observe an association between the total lymphocyte count and limitation in functional capacity $(\chi 2=6.94 ; p=0.008$; OR: 5.23; 95\% Cl: 1.441-19.025). Conclusions: Malnutrition in patients with digestive tract cancer was associated with limitation in functional capacity.
\end{abstract}

KEY WORDS: Subjective global assessment. Malnutrition. Karnofsky index. Functional capacity. Cancer.

\section{Introduction}

Cancer is a chronic, disabling disease with high mortality. The World Health organization (WHO) estimates that, by the year of 2030, around 12 million people will die owing to this condition ${ }^{1}$, with gastrointestinal (Gl) tract tumors standing out ${ }^{2}$. In Mexico, changes in alimentary habits have walked hand in hand with an increase of these neoplasms, out of which gastric cancer is at first place, followed by colon and rectum cancer ${ }^{3}$.

Malnourishment in the cancer patient results from multiple factors that are often associated with anorexia, cachexia and early satiety sensation, which are often experienced by individuals with cancer. Both are closely related, so that the disease can cause malnourishment and this, in turn, can negatively influence on the disease ${ }^{4}$. If malnourishment is not opportunely treated, it can entail increasing emaciation, weakness, reduced protein synthesis and loss of muscular mass, whereby it directly affects the cancer patient quality of life and increases mortality ${ }^{5-7}$.

There are different tools designed for nutritional screening and evaluation. The Subjective Global Assessment (SGA), developed in the 1980's at the Toronto General Hospital, has been shown to be a highly reliable and easily reproducible tool, with even higher sensitivity and specificity than traditional parameters such as albumin and transferrin values to assess the nutritional status ${ }^{8,9}$. The Karnofsky index (KI) was first used in the decade of 1950 to assess the performance status in cancer patients undergoing chemotherapy. Ever since, it has been used in different clinical trials to assess the functional capacity, as a predictor of

\footnotetext{
Correspondence:

Elizabeth Pérez Cruz

Av. Instituto Politécnico Nacional \#5160, Magdalena de las Salinas C.P. 07760, Ciudad de México, México E-mail: pece_liz@ hotmail.com
}

Gac Med Mex. 2017;153:523-527

Contents available at PubMed www.gacetamedicademexico.com 
evolution, survival and even as a quality of life indicator ${ }^{10-12}$.

The KI score has been associated with the Quetelet index (QI) in cancer patients, with a survival increase being observed the higher the latter is ${ }^{13}$. Some studies show an association between malnourishment and the occurrence of complications associated with changes in functional capability. Weight loss has also been described as a poor prognosis indicator in these patients, with it being related to functional capability reduction, which suggests a possible direct relationship with the presence of malnourishment ${ }^{2,3}$. The purpose of this study was to find out the nutritional status and its association with functional capacity in patients with Gl cancer.

\section{Methods}

This was a retrospective, analytical, observational study carried out in adult patients admitted to the oncology hospitalization area of a federal reference hospital of Mexico City during the years 2011 and 2012. Patients from both genders, with a Gl tract cancer initial diagnosis; i.e., less than one year since the onset of symptoms at diagnosis and no previous oncologic treatment, were included. Patients with incomplete data were excluded.

Study variables were the nutritional status and functional capacity, assessed at patient admission. Anthropometrics, albumin and total lymphocyte count were also determined. Demographic variables were age, gender, level of education, smoking, alcoholism and comorbidity.

Nutritional status was determined by means of the SGA, which was obtained from the records of the nutritional support unit, since SGA is systematically applied to all patients within 24-72 hours of admission. This method combines patient history aspects (weight loss, changes in alimentary intake and changes in functional capacity) and physical examination findings (loss of fat and muscle mass, presence of sacral edema or ascites). Patients were classified in three different nutritional situations: well-nourished, moderate malnourishment and severe malnourishment ${ }^{14}$.

Functional capacity was measured by means of the $\mathrm{KI}$. Scores of the scale range from 0 to 100 , where a high score means that the patient has better capacity to perform daily activities (10: moribund patient; 100: patient able to lead a normal life, independent and without assistance). Patients were classified in two groups: without functional limitation $(\mathrm{KI} \geq 80)$ and with functional limitation $(\mathrm{KI} \leq 70)^{10,11}$.

Recorded anthropometric measurements included height $(\mathrm{cm})$ and weight $(\mathrm{kg}), \mathrm{QI}\left(\mathrm{kg} / \mathrm{m}^{2}\right)$ and weight loss in the previous 6 months, which was calculated with the formula:

$$
\text { Weight loss }(\%)=\frac{\left(\begin{array}{l}
\text { Current weight }- \\
\text { usual weight }
\end{array}\right) \times 100}{\text { Usual weight }}
$$

Nutritional biochemical and immunological parameters complemented the nutritional assessment and were obtained from patient records. Considering albumin values, patients were classified as: no malnourishment (> $3.5 \mathrm{~g} / \mathrm{dL}$ ), with mild malnourishment (3.5-2.8 g/dL), with moderate malnourishment (2.1$2.7 \mathrm{~g} / \mathrm{dL})$ and with severe malnourishment $(<2.1 \mathrm{~g} / \mathrm{dL})$. The cutoff points considered for classification according to lymphocyte total count [(lymphocyte \% x leukocyte)/100] were $>1500 \mathrm{~mm}^{3}$ for normal, 1200-1500 $\mathrm{mm}^{3}$ for mild malnourishment, $800-1199 \mathrm{~mm}^{3}$ for moderate malnourishment and $<800 \mathrm{~mm}^{3}$ for severe malnourishment.

Data analysis was carried out using the SPSS statistical package, version 14.0 (SPSS Inc., Chicago, IL, USA). Descriptive statistics was used. Qualitative variables were analyzed by means of frequencies and percentages; quantitative variables, with means and standard deviations. The tests used to analyze the association between nutritional status and functionality were the odds ratio and the chi-square test. The level of significance adopted for comparisons was $\mathrm{a}=$ $5 \%$. The study was approved by the Ethics and Research Committee with institutional registry number HJM2013/11R.

\section{Results}

Fifty-seven GI tract cancer-diagnosed patients were finally evaluated. Average age was $57.8 \pm 14.5$ years, with $54 \%$ being men and $46 \%$ women. Weight loss was referred by $96 \%$ of patients, and it was estimated at an average of $13.18 \pm 8.73 \%$. A weight loss of $11-20 \%$ from usual weight was experienced by $35 \%$ of the population, with $17 \%$ of the population referring weight loss of $20-30 \%$ and $34 \%$ having a weight loss of $1-10 \%$; only $4 \%$ of the study population had no weight loss recorded. Demographic characteristics are shown in table 1. 
Table 1. Demographic characterization of the study population

\begin{tabular}{lccc}
\hline Characteristic & Global $(\mathbf{n}=\mathbf{5 7})$ & No malnourishment $(\mathbf{n}=\mathbf{1 0})$ & With malnourishment $(\mathbf{n}=\mathbf{4 7})$ \\
\hline Age (years) & $57.8 \pm 14.5$ & $54.9 \pm 5.71$ & $58.43 \pm 14.4$ \\
Gender (M/F) & $31 / 26$ & $4 / 6$ & $27 / 20$ \\
Weight loss (\%) & $13.18 \pm 8.73$ & $5.9 \pm 6.9$ & $14.7 \pm 8.33$ \\
Ql (kg/m²) & $22.6 \pm 3.8$ & $21.3 \pm 3.4$ & $22.8 \pm 3.8$ \\
Active smoking & $27(47.4 \%)$ & $7(70 \%)$ & $20(42.5 \%)$ \\
\hline
\end{tabular}

QI: Querelet Index.

Predominating types of cancer were colon (35\%), gastric $(23 \%)$, pancreas $(9 \%)$, esophagus $(7 \%)$, small bowel $(2 \%)$ and others (24\%). Most patients (94.8\%) were at clinical stages II and III of disease; only $1.7 \%$ was at clinical stage I and $3.5 \%$ at clinical stage II.

Malnourishment, as measured by SGA, affected $82.5 \%$ of the population, out of which $21 \%$ had moderate malnourishment and $61.5 \%$ severe malnourishment. By biochemical parameters, such as albumin, $18 \%$ was classified within normal, $24 \%$ with mild, $21 \%$ with moderate and $37 \%$ with severe depletion, with an average of $3.2 \pm 0.79 \mathrm{~g} / \mathrm{dL}$. When immunological parameters were considered, such as lymphocyte total count, $65 \%$ had malnourishment: had $14 \%$ mild malnourishment, $21 \%$ moderate malnourishment and $30 \%$ severe malnourishment.

With regard to $\mathrm{KI}$-determined functional capacity, $24.5 \%$ of the population was carrying out its activities practically normally, whereas $75.5 \%$ had some activity limitation. When a sub-analysis as an overall indicator of self-sufficiency was made, out of that $75.5 \%$, we found that $35 \%$ had some limitations, with scores of $80 ; 35 \%$ had limited activity, i.e., had scores ranging from 50 to 70 ; and $5.5 \%$ were unable of self-care and had scores ranging from 10 to 40 .

The association between nutritional status by SGA and functional capacity of the population is shown in table 2. Of the patients who showed malnourishment, $78.7 \%$ had activity limitations $\left(\chi^{2}=1.56 ; p=0.212\right)$. Table 3 shows the association between nutritional status, classified by lymphocyte total count, and functional capacity. Of those who showed malnourishment, $56.1 \%$ had activity limitations $\left(\chi^{2}=6.94\right.$; $p=0.008)$.

Table 4 shows the relationships between functional capacity and study predictor variables.

\section{Discussion}

Malnourishment in cancer patients is a reality, and weight loss has been an indicator thereof. Some
Table 2. Association between nutritional status by SGA and functional capacity according to KI

\begin{tabular}{lccc}
\hline $\begin{array}{l}\text { Functional } \\
\text { capacity }\end{array}$ & \multicolumn{2}{c}{ Nutritional status } & Total \\
\cline { 2 - 3 } & $\begin{array}{c}\text { No } \\
\text { malnourishment }\end{array}$ & $\begin{array}{c}\text { With } \\
\text { malnourishment }\end{array}$ & \\
\hline Not limited & 4 & 10 & 14 \\
Limited & 6 & 37 & 43 \\
Total & 10 & 47 & 57 \\
\hline$x^{2}=1.56(p=0.212)$. & & \\
OR: $2.46(95 \% \mathrm{Cl}: 0.581-10.465)$. & &
\end{tabular}

Table 3. Association between nutritional status by immunology tests (lymphocyte total count) and functional capacity according to $\mathbf{K I}$

\begin{tabular}{lccc}
\hline $\begin{array}{l}\text { Functional } \\
\text { capacity }\end{array}$ & \multicolumn{2}{c}{ Nutritional status } & Total \\
\cline { 2 - 3 } & $\begin{array}{c}\text { No } \\
\text { malnourishment }\end{array}$ & $\begin{array}{c}\text { With } \\
\text { malnourishment }\end{array}$ & \\
\hline Not limited & 9 & 5 & 14 \\
Limited & 11 & 32 & 43 \\
Total & 20 & 37 & 57 \\
\hline$x^{2}=694(p=00008)$ & & &
\end{tabular}

$x^{2}=6.94(p=0.008)$

OR: 5.23 (95\% Cl: 1.441-19.025).

Table 4. Relationships between functional capacity and biochemical and clinical variables

\begin{tabular}{lcc}
\hline Característica & $\mathbf{r}$ & $\mathbf{p}$ \\
\hline Cholesterol & 0.174 & 0.490 \\
Albumin & 0.400 & 0.826 \\
Lymphocyte & 0.255 & 0.055 \\
Cancer stage & 0.489 & 0.001 \\
\hline
\end{tabular}

studies have shown that, at diagnosis, $80 \%$ of patients with $\mathrm{Gl}$ cancer have already experienced significant weight loss, generally of at least $10 \%$ of body weight over a period of 6 months ${ }^{4,5,15}$. In our study population, we reported a higher percentage of patients with weight loss, with average losses higher than $10 \%$ in 6 months. Most part of our patients had colon cancer, 
followed by gastric cancer, which may account for this higher weight loss. This, reinforced by findings of Moon et al. ${ }^{16}$, together with reduced intake, dysphagia and mucositis that are even accentuated during cancer treatments, mainly with chemotherapy ${ }^{17}$.

When patients were assessed my means of the QI, we observed that although more than half had a normal or even higher index, when SGA is applied, we see that more than two thirds of the population had some degree of malnourishment. Tolentino et al. ${ }^{18}$ reported, in their study in cancer patients, that although weight loss is quite common, QI decreased mainly in the presence of GI tumors in comparison with non-GI tumors. In our study, we determined that, although QI was similar in both groups, weight loss was higher in the group with SGA-determined malnourishment. Therefore, $\mathrm{QI}$ is not a sensitive indicator to detect malnourishment. The use of SGA continues to be a practical method that enables to distinguish between well-nourished and malnourished patients, without the need to resort to anthropometric or laboratory measurements.

Malnourishment, as a deficiency of multiple nutrients, alters functions that the immune system is implied with. For this purpose, the determination of total leukocyte and different white cell numbers is a simple procedure that allows for useful information to be obtained and that has been associated with cancer porognosis ${ }^{19-21}$. Even neutrophil/lymphocyte and platelet/ lymphocyte ratios have been studied as predictors of both aggressiveness and poor prognosis, since neutrophils are a source of endothelial growth factor, which participates in angiogenesis and increases the capability of the tumor to spread. In addition, elevation of systemic inflammation markers is associated with lymphocytopenia and T-cell altered anti-tumor response $\mathrm{e}^{22-24}$.

Considering that immune system cell functions are influenced by antioxidant and pro-oxidant substances, and that cancer cells undergo disturbances in electron energy balance, we determined leukocyte total values as a malnourishment marker and observed that more than half the patients had some degree of malnutrition, in addition to finding a clear association with functional capacity, which is something we didn't verify in other biochemical biomarkers such as albumin or cholesterol.

Nutritional status alterations are worrisome in the cancer patient, especially malnourishment, since different studies have associated it with tolerance to treatments, quality of life and survival. To assess nutritional status impact and its possible association with functional capacity, we used the KI. Although there are different scales to measure functional capacity, the KI has been classified as the best method owing to its survival predicting power, which enables to determine the level of activity and dependence of patients with regard to their medical care ${ }^{25,26}$. The Eastern Cooperative Oncology Group (ECOG)/WHO Functional Scale, first applied in 1960 to patients receiving chemotherapy, has shown high correlation with KI. Spearman correlation coefficient-obtained values were $0.85(p>0.0001)$ in the study by Loperinzi et al. ${ }^{26}$, and $0.87(p>0.0001)$ in the study by Buccheri et al. ${ }^{27}$. The Palliative Care Functional Scale is a functional capacity measuring scale specifically designed for patients on palliative care, just as Edmonton Functional Assessment Tool. The Palliative Care Functional Scale is a modification of $\mathrm{KI}$ and, therefore, is has high correlation (Spearman coefficient of 0.94). In our study we observed that, although $\mathrm{KI}$ values are not as low as those reported in other series, patients already have some degree of limitation and certain dependence; thus, only $5.5 \%$ had a $\mathrm{KI}<50$, with expected survival of less than 6 months $^{28,29}$. In addition, we observed an association between nutritional status by SGA and functional capacity by $\mathrm{KI}$. This is related to the fact that malnourishment associated with the disease, with its stage, and even with the treatments received by the patient, produces a decrease in fat mass and muscular strength, which have an immediate effect of physical activity and functional capacity reduction in these patients. Ottery ${ }^{30}$, in a study of patients with Gl cancer, identified that nutritional status, disease evolution and tumor location were independent factors on functionality and quality of life.

The scales to assess functional capacity have shown its survival predictive power, which can be attributed to clinical estimates. Although Maltoni et al ${ }^{1}$ have called this claim into question, in our study we observed a negative correlation between functional capacity and cancer clinical stage, so that it depends on patient evolution, and the more advanced the disease is, the better the prognostic estimate based on functional capacity measurement will be.

Baseline nutritional status identification and nutritional intervention influence on antineoplastic treatment success and general prognosis of a cancer patient. Although nutritional support contributes to normalize nutritional status, improve functionality and quality of life, the therapeutic approach should not be 
forgotten in those patients at early and locally advanced stages, whereas in those at advanced or metastatic stages, focus should be on symptom control, quality of life improvement and overall survival increase i2-34. $^{32}$.

Finally, it is necessary for our centers to have nutritional assessment tools available, which enable opportune identification of those patients at risk of or with malnourishment; in addition, having a performance status and quality of life assessment scale is also required, since it is indispensable from the therapeutic point of view according to its purposes.

\section{Conclusions}

The prevalence of malnutrition in our setting is high in patients with Gl cancer. Nutritional status determined by SGA and by total leukocyte count is associated with functional capacity.

\section{Conflicts of interests}

There are no conflicts of interests. There is no economic, personal, political or academic relationship, nor have benefits of money, goods, hospitality or subsidies been received from any source that might influence on the presentation of the results of this study.

\section{References}

1. American Cancer Society. Cancer facts and figures 2011. Atlanta: American Cancer Society; 2011. p. 1-55.

2. McMahon K, Decker G, Ottery FD. Integrating proactive nutritional assessment in clinical practices to prevent complications and cost. Semin Oncol. 1998:25(2 Suppl 6):20-7.

3. Abdo Francis JM. Cáncer del aparato digestivo. Revista Médica Hospital General de México. 2010;73:7.

4. Bruera E. ABC of palliative care. Anorexia, cachexia, and nutrition. BMJ. 1997;315:1219-22.

5. Van Cutsem E, Arends J. The causes and consequences of cancer-associated malnutrition. Eur J Oncol Nurs. 2005;9(Suppl 2):S51-63.

6. McMillan DC. An inflammation-based prognostic score and its role in the nutrition-based management of patients with cancer. Proc Nutr Soc. 2008;67:257-62.

7. Eldridge B, Rock CL, McCallum PD. Nutrition and the Patient with Cancer. In: Nutrition in the Prevention and Treatment of Disease. Ed. Coulston AM, Rock CL, Monsen ER. Academic Press, San Diego, 2001. pg. 397-412.

8. Ek AC, Unosson M, Larsson J, et al. Interrater variability and validity in subjective nutritional assessment of elderly patients. Scand J Caring Sci. 1996;10:163-8.

9. Enia G, Sicuso C, Alati G, et al. Subjective Global Assessment of nutrition in dialysis patients. Nephrol Dial Transplant.1993;8:1094-8.

10. Karnofsky DA, Abelman WH, Craver LF, et al. The use of nitrogen mustards in the palliative treatment of cancer. Cancer. 1948;1:634-56.
11. Karnofsky DA, Burchenal JH. The clinical evaluation of chemotherapeutic agents in cancer. En: MacLeod CM, editor. Evaluation of chemotherapeutic agents. New York: Columbia University Press; 1949. p. 191-205.

12. Puiggròs $C$, Lecha $M$, Rodríguez $T$, et al. Karnofsky Index as a mortality predicting factor in patients on home-based enteral nutrition. Nutr Hosp. 2009;24:156-60.

13. Ruiz-Delgado GJ, Lutz-Presno JA, Alarcón-Urdaneta C, et al. Body mass index as an indicator of prognosis in patients undergoing allogenic hematopoietic stem cell transplantation. Rev Hematol Mex. 2011;12:28-31.

14. Detsky AS, McLaughlin JR, Baker JP, et al. What is subjective global assessment of nutritional status? JPEN J Parenter Enteral Nutr. 1987:11:8-13.

15. Rivadeneira DE, Evoy D, Fahey TJ 3rd, et al. Nutritional support of the cancer patient. CA Cancer J Clin. 1998;48:69-80.

16. Moon $\mathrm{H}$, Roh JL, Lee SW, et al. Prognostic value of nutritional and hematologic markers in head and neck squamous cell carcinoma treated by chemoradiotherapy. Radiother Oncol. 2016;118:330-4.

17. Cho YW, Roh JL, Jung JH, et al. Prediction of prosttreatment significant body weight loss and its correlation with disease free survival in patients with oral squamous cell carcinomas. Nutr Cancer. 2013;65:417-23.

18. Tolentino R, Quizon O, Llido L. Nutritional status of patients with malignancy of the gastrointestinal tract and other malignancies - comparison of characteristics and pattern: a two-year study in a private tertiary care hospital in the Philippines. Philip J Oncology. 2007;8:37-44.

19. Huang SH, Waldron JN, Milosevic M, et al. Prognostic value of pretreatment circulating neutrophils, monocytes, and lymphocytes in oropharyngeal cancer stratified by human papillomavirus status. Cancer. 2015;121:545-55.

20. De Carvalho TM, Miguel Marin D, da Silva CA, et al. Evaluation of patients with head and neck cancer performing standard treatment in relation to body composition, resting metabolic rate, and inflammatory cytokines. Head Neck. 2015;37:97-102.

21. Rassouli A, Saliba J, Castano R, et al. Systemic inflammatory markers as independent prognosticators of head and neck squamous cell carcinoma. Head Neck. 2015;37:103-10.

22. Cannon NA, Meyes J, lyengar P, et al. Neutrophil-lymphocyte and platelet-lymphocyte ratios as prognostic factors after stereotactic radiation therapy for early-stage non-small-cell lung cancer. J Thorac Oncol. 2015;10:280-5.

23. Fan W, Zhang Y, Wang Y, et al. Neutrophil-to-lymphocyte and platelet-to-lymphocyte ratios as predictors of survival and metastasis for recurrent hepatocellular carcinoma after transarterial chemoembolization. PLos One. 2015;10:e0119312.

24. Kim EY, Lee JW, Yoo HMY. The platelet-to-lymphocyte ratio versus neutrophil-to-lymphocyte ratio: which is better as a prognostic factor in gastric cancer? Ann Surg Oncol. 2015:22:4363-70.

25. Mor V, Laliberte L, Morris JN, et al. The Karnofsky Performance Status Scale: an examination of its reliability and validity in a research setting. Cancer. 1984;53:2002-7.

26. Loperinzi CL, Laurie JA, Wieand HS, et al. Prospective evaluation of prognostic variables from patient-completed questionnaires. J Clin Oncol. 1994;12:601-7.

27. Buccheri G, Ferrigno D, Tamburini M. Karnofsky and ECOG performance status scoring in lung cancer: a prospective, longitudinal study of 536 patients from a single institution. Eur $\mathrm{J}$ Cancer. 1996;32A:1531-41.

28. Yates JW, Chalmer B, McKegney FP. Evaluation of patients with advanced cancer using the Karnofsky Performance Status. Cancer. 1980;45:2220-4.

29. Maltoni M, Pirovano M, Scarpi E, et al. Prediction of survival of patients terminally ill with cancer. Results of an Italian prospective multicentric study. Cancer. 1995;75:2613-22.

30. Ottery FD. Supportive nutrition to prevent cachexia and improve quality of life. Semin Oncol. 1995;22(Suppl 3):98-111.

31. Maltoni M, Nanni O, Derni S, et al. Clinical prediction of survival is more accurate than the Karnofsky performance status in estimating life span of the terminally ill cancer patients. Eur J Cancer. 1994;30:764-6.

32. McMillan DC. An inflammation-based prognostic score and its role in the nutrition-based management of patients with cancer. Proc Nutr Soc. 2008;67:257-62.

33. Gârtner $\mathrm{S}$, Krûger J, Aghdassi AA, et al. Nutrition in pancreatic cancer: a review. Gastrointest Tumors. 2015;2:195-202.

34. Rosania R, Chiapponi C, Malfertheiner $P$, et al. Nutrition in patients with gastric cancer: an update. Gastrointest Tumors. 2015;2:178-87. 